\title{
Fluctuations, Classical Activation, Quantum Tunneling, and Phase Transitions
}

\author{
D. L. Stein \\ Departments of Physics and Mathematics, University of Arizona, Tucson, AZ 85721 USA
}

Received on 20 November, 2004

\begin{abstract}
We study two broad classes of physically dissimilar problems, each corresponding to stochastically driven escape from a potential well. The first class, often used to model noise-induced order parameter reversal, comprises Ginzburg-Landau-type field theories defined on finite intervals, perturbed by thermal or other classical spatiotemporal noise. The second class comprises systems in which a single degree of freedom is perturbed by both thermal and quantum noise. Each class possesses a transition in its escape behavior, at a critical value of interval length and temperature, respectively. It is shown that there exists a mapping from one class of problems to the other, and that their respective transitions can be understood within a unified theoretical context. We consider two applications within the first class: thermally induced breakup of monovalent metallic nanowires, and stochastic reversal of magnetization in thin ferromagnetic annuli. Finally, we explore the depth of the analogy between the two classes of problems, and discuss to what extent each case exhibits the characteristic signs of critical behavior at a sharp second-order phase transition.
\end{abstract}

\section{INTRODUCTION}

Noise-induced escape from a locally stable state governs a wide range of physical phenomena [1]. In spatially extended classical systems, where the noise is typically, but not necessarily, of thermal origin, these phenomena include homogeneous nucleation of one phase inside another [2], micromagnetic domain reversal [3, 4], pattern formation in nonequilibrium systems [5], and many others. The concepts and techniques employed are formally identical to those used in tunneling problems in quantum field theories, including among others the 'decay of the false vacuum' [6], anomalous particle production [7], and macroscopic quantum tunneling in a dc SQUID [8].

Recently a new kind of 'phase transition', in the classical activation behavior of spatially extended systems perturbed by weak spatiotemporal noise, has been uncovered and analyzed [9-11]. The existence of a similar crossover, from thermal activation to quantum tunneling in systems as simple as a single degree of freedom, has long been known [12-22]. Here we will review the stochastic escape behaviors of both types of systems, explore the depth of the analogy between them, discuss to what extent the crossover can be thought of as a type of second-order phase transition, and show how the purely classical transition, occurring as system length is varied, can shed light on the crossover from classical activation to quantum tunneling as temperature is lowered. But let's begin with a purely classical problem.

\section{MODEL}

Consider an extended system described by a classical field $\phi(z, t)$ defined on the spatial interval $[-L / 2, L / 2]$, subject to the potential $V(\phi)$ and perturbed by spatiotemporal white noise. Its time evolution is governed by the stochastic Ginzburg-Landau equation

$$
\partial_{t} \phi=\partial_{z z} \phi-\partial_{\phi} V(\phi)+\sqrt{2 T} \xi(z, t)
$$

where all dimensional quantities have been scaled out. The first term on the RHS arises from a field 'stiffness', i.e., an energy penalty for spatial variation of the field. The system is stochastically perturbed by additive white noise $\xi(z, t)$, satisfying $\left\langle\xi\left(z_{1}, t_{1}\right) \xi\left(z_{2}, t_{2}\right)\right\rangle=\delta\left(z_{1}-z_{2}\right) \delta\left(t_{1}-t_{2}\right)$. We consider only weak noise, i.e., the noise magnitude $T$ is small compared to all other energy scales in the problem (formally, our analysis will be asymptotically valid in the $T \rightarrow 0$ limit). The frictional coefficient has been set to one, so when $T$ is temperature Eq. (1) obeys the fluctuation-dissipation relation.

The deterministic, or zero-noise, dynamics can be written as the variation of an action $\mathcal{H}$ with the field $\phi$ :

$$
\dot{\phi}=-\delta \mathcal{H} / \delta \phi,
$$

and because the dynamics is gradient, the action $\mathcal{H}$ is simply an energy functional

$$
\mathcal{H}[\phi] \equiv \int_{-L / 2}^{L / 2} d z\left[\frac{1}{2}\left(\partial_{z} \phi\right)^{2}+V(\phi)\right] .
$$

We will mostly consider relatively simple potentials, in particular the symmetric bistable quartic potential

$$
V_{s}(\phi)=-\frac{1}{2} \phi^{2}+\frac{1}{4} \phi^{4},
$$

and the asymmetric monostable cubic potential

$$
V_{a}(\phi)=\frac{1}{2} \phi^{2}-\frac{1}{3} \phi^{3} \text {. }
$$

Both potentials are shown in Fig. 1.

In the weak-noise $(T \rightarrow 0)$ limit, the classical activation rate for a transition out of a stable well is

$$
\Gamma \sim \Gamma_{0} \exp (-\Delta E / T),
$$

where $\Delta E$ is the activation barrier (to be defined below) and $\Gamma_{0}$ the rate prefactor, determined by fluctuations about the most probable escape path. When the top of the barrier is locally quadratic, the prefactor $\Gamma_{0}$ is independent of temperature. In such circumstances the escape rate (6) is said to be of the Arrhenius-van't Hoff (or often simply Arrhenius) form. 


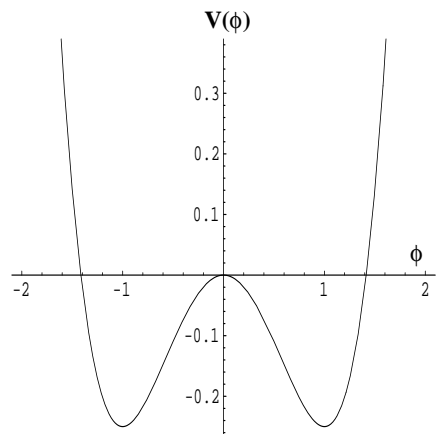

(a)

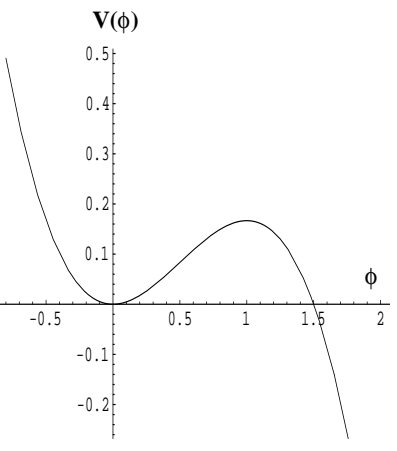

(b)
FIG. 1: Potentials used in discussion: (a) bistable quartic (Eq. (4)) and (b) monostable cubic (Eq. (5)).

\section{THE INFINITE-DOMAIN CASE}

Let's start by considering the $L=\infty$ case, which was fully worked out for the classical nucleation problem by Langer [2] and for the quantum tunneling problem by Callan and Coleman [6]. (See also Schulman [23] for a good pedagogic treatment.) For illustrative purposes, we will use the bistable quartic potential (4).

The states of interest - i.e., the stable and saddle field configurations - are time-independent solutions of the zeronoise Ginzburg-Landau equation $\dot{\phi}=-\delta \mathcal{H} / \delta \phi$. They are therefore extrema of the action so in the current case satisfy the nonlinear differential equation

$$
\phi^{\prime \prime}=-\phi+\phi^{3} .
$$

Its spatially uniform solutions are just the stable states $\phi(z)=$ \pm 1 and the local maximum $\phi=0$. But our main interest is in the soliton-like pair (or 'bounce' [6])

$$
\phi(z)= \pm \tanh \left[\left(z-z_{0}\right) / \sqrt{2}\right]
$$

where $z_{0}$ is a constant whose presence denotes the fact that the 'domain wall', i.e. the spatially varying piece of (8) that separates the two stable states, can nucleate anywhere on the line.

Assuming for the moment that (8) is the saddle configuration (this will be justified below), the activation energy $\Delta E=E\left[\phi_{\text {saddle }}\right]-E\left[\phi_{\text {stable }}\right]$ in the Kramers rate formula (6) can be computed, giving

$$
\begin{array}{r}
\Delta E=\int_{-\infty}^{\infty} d z\left[\frac{1}{2}\left(\partial_{z} \phi_{\text {saddle }}\right)^{2}+V\left(\phi_{\text {saddle }}\right)\right] \\
-\int_{-\infty}^{\infty} d z\left[\frac{1}{2}\left(\partial_{z} \phi_{\text {stable }}\right)^{2}+V\left(\phi_{\text {stable }}\right)\right]=\frac{2 \sqrt{2}}{3}
\end{array}
$$

which is essentially the energy of a single domain wall.

In order to compute the prefactor $\Gamma_{0}$ of the Kramers rate (6), we need to examine fluctuations about the optimal escape path, in particular in the vicinity of the stable and saddle field

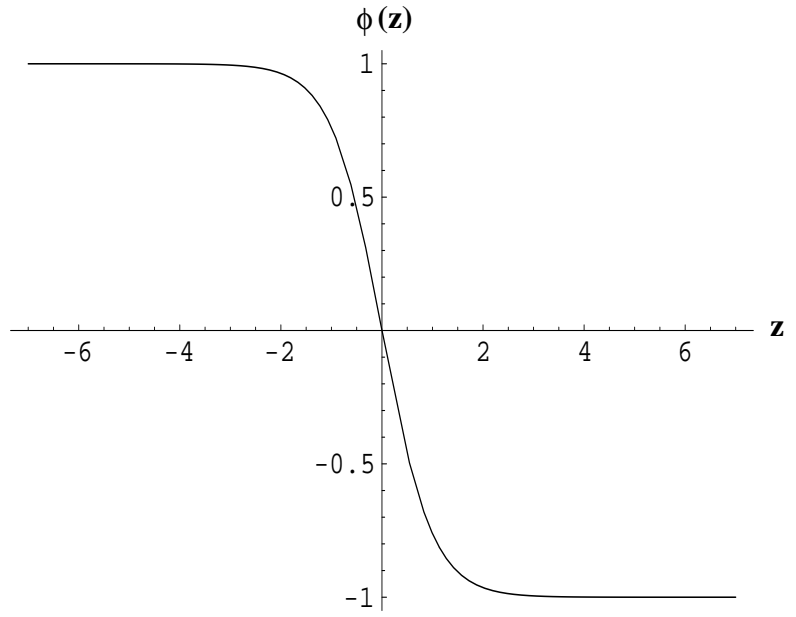

FIG. 2: The 'bounce' described by Eq. (8) with $z_{0}=0$. For clarity, only one of the symmetric pair is shown.

configurations. The procedure for doing this is described in detail elsewhere (see, for example, $[11,23,24])$, and will be simply summarized here.

Let $\varphi_{s}$ denote the stable state (in this case, the uniform \pm 1 state), and let $\varphi_{t}$ denote the transition (saddle) state (here given by Eq. (8)). Consider a small perturbation $\eta$ about the stable state, i.e., $\varphi=\varphi_{s}+\eta$. Then to leading order $\dot{\eta}=-\Lambda_{s} \eta$, where $\Lambda_{s}$ is the linearized zero-noise dynamical operator governing the time evolution of fluctuations about $\varphi_{s}$. Similarly $\Lambda_{t}$ is the linearized zero-noise dynamical operator for $\varphi_{t}$.

We next diagonalize the linear time-evolution operators, by decomposing fluctuations about the stable and transition states into normal modes, which are eigenfunctions $\eta_{i}$ of the corresponding operators:

$$
\Lambda_{b} \eta_{i}=\lambda_{i} \eta_{i}
$$

where $b=s, t$. An eigenfunction with positive eigenvalue $\lambda_{i}>$ 0 is a stable mode; one with $\lambda_{i}<0$ is unstable. A stable (or metastable) state therefore has all $\lambda_{i}>0$; a saddle state has a single $\lambda_{i}<0$. Its corresponding eigenfunction denotes the unstable direction (in function space) in the vicinity of the saddle, leading either back to the initial stable configuration or out of the well entirely. With the potential (4), the eigenvalue equation becomes

$$
\dot{\eta}=-\Lambda_{b} \eta \equiv-\left[-d^{2} / d z^{2}+\left(-1+3 \phi_{b}^{2}\right)\right] \eta .
$$

In most cases the barrier is locally quadratic: all eigenvalues are nonzero and we're left with an infinite set of decoupled quadratic fluctuations about the stable and saddle states. Then $[24,25]$

$$
\Gamma_{0}=\frac{1}{2 \pi} \sqrt{\left|\frac{\operatorname{det} \Lambda_{s}}{\operatorname{det} \Lambda_{t}}\right|}\left|\lambda_{t, 0}\right|,
$$

where $\lambda_{t, 0}$ is the only negative eigenvalue of $\Lambda_{t}$. In general, the determinants in (12) separately diverge: they are products 
of an infinite number of eigenvalues with magnitude greater than one. However, their ratio, which can be interpreted as the limit of a product of individual eigenvalue quotients, is finite.

There is a technical difficulty that has not yet been addressed, namely the existence of a soft collective mode corresponding to the arbitrariness of $z_{0}$ in (8): the domain wall (or 'instanton') can nucleate anywhere. The resulting translational symmetry implies a zero-eigenvalue mode. Its removal can be achieved with the McKane-Tarlie regularization procedure [26] (see also [27]) for functional determinants. This will not be discussed further here, except to note that its presence results, among other things, in a non-Arrhenius (noisedependent) prefactor that scales with the length. The overall result for the prefactor per unit length is then

$$
\Gamma_{0} / L=\left(\frac{4 \sqrt{6}}{\pi}\right)\left(\frac{2}{\sqrt{\pi T}}\right)
$$

where the first term on the RHS follows from the computation of Eq. (12) with the zero eigenvalue removed via the McKaneTarlie procedure, and the second term (divided through by length $L$ ) gives the contribution of the zero eigenvalue, i.e., the effect of the translational symmetry of the bounce.

\section{THE FINITE-DOMAIN CASE}

The differences between the infinite and finite-line cases are not only quantitative; there are important qualitative differences that are also relevant, in an entirely different context, to the classical $\leftrightarrow$ quantum crossover (Sec. VI). The most striking of these differences is a sharp change in activation behavior as interval length is varied; moreover, this change exhibits characteristics of a second-order phase transition [9$11]$, but only in a strictly asymptotic sense.

Because we are now working on an interval of finite length, we need to specify boundary conditions. With the exception of the zero-energy mode that accompanies only translationinvariant (such as periodic) boundary conditions, different choices of boundary conditions lead only to minor quantitative differences. To avoid the (minor) complication of the zero mode altogether, we will employ Neumann boundary conditions: $\partial \phi /\left.\partial L\right|_{-L / 2}=\partial \phi /\left.\partial L\right|_{L / 2}=0$. With this choice $\phi_{\text {stable }}= \pm 1$, as before. We will continue to use the notation of Sec. III, where $\phi_{s}\left(\phi_{t}\right)$ refers to the stable (transition) state.

For the symmetric $\phi^{4}$ potential with Neumann boundary conditions, the change in activation behavior arises from a bifurcation of the transition state, from a uniform configuration below a critical length $L_{c}$ to a pair of degenerate, spatially varying 'bounce' configurations above $L_{c}$. More precisely, the saddle states are

$$
\phi_{t}=0
$$

when $L<L_{c}$ and

$$
\phi_{t}= \pm \sqrt{\frac{2 m}{1+m}} \operatorname{sn}\left(\frac{x}{\sqrt{m+1}} \mid m\right)
$$

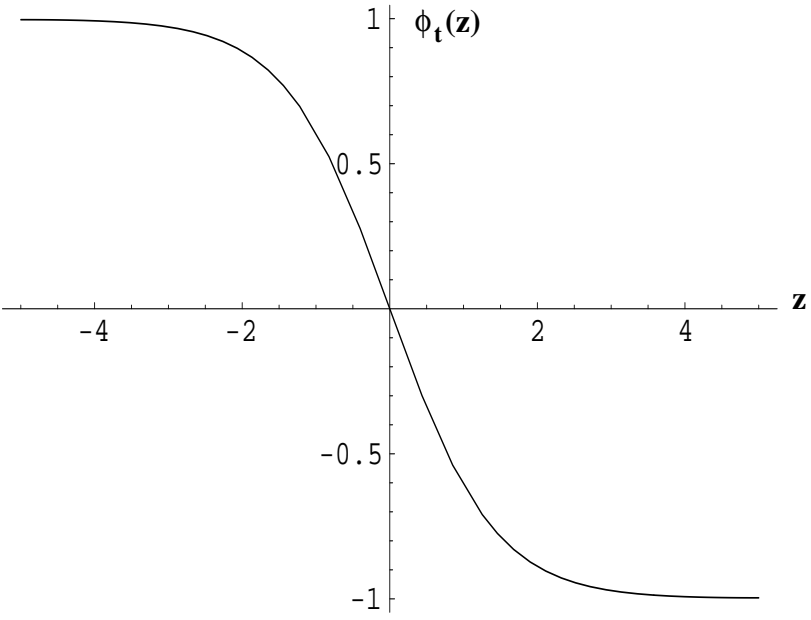

FIG. 3: The transition state $\phi_{t}(z)$ for $L=10$ (corresponding to $m=$ 0.986 ) described by Eq. (15). As in Fig. 2, only one of the symmetric pair is shown.

when $L \geq L_{c}$, where $\operatorname{sn}(\cdot \mid m)$ is the Jacobi elliptic sn function with parameter $0 \leq m \leq 1$. Its quarter-period is given by $\mathbf{K}(m)$, the complete elliptic integral of the first kind [28], which is a monotonically increasing function of $m$. As $m \rightarrow$ $0^{+}, \mathbf{K}(m)$ decreases to $\pi / 2$, and $\operatorname{sn}(\cdot \mid m) \rightarrow \sin (\cdot)$. In this limit the saddle state smoothly degenerates to the $\phi=0$ configuration. As $m \rightarrow 1^{-}$, the quarter-period increases to infinity (with a logarithmic divergence), and $\operatorname{sn}(\cdot \mid m) \rightarrow \tanh (\cdot)$, the (nonperiodic) single-kink sigmoidal function. The Langer/CallanColeman bounce solution (8) is thereby recovered as $L \rightarrow \infty$.

The value of $m$ in (15) is determined by the interval length $L$ and the Neumann boundary conditions, which require that

$$
L / \sqrt{m+1}=2 \mathbf{K}(m) .
$$

The critical length is determined by (16) when $m=0$; that is, $L_{c}=\pi$. As previously noted, $m \rightarrow 1$ corresponds to $L \rightarrow \infty$, and the activation energy smoothly approaches the asymptotic value of $2 \sqrt{2} / 3$. The transition state for an intermediate value of $m$, corresponding to $L=10$, is shown in Fig. 3 .

The activation energy $\Delta E$ can be solved in closed form for all $L>L_{c}$ (below $L_{c}$, it is simply $L / 4$ ):

$\Delta E=\frac{1}{3(1+m)^{3 / 2}}\left[4(1+m) \mathbf{E}(m)-\frac{1}{2}(1-m)(3 m+5) \mathbf{K}(m)\right]$,

with $\mathbf{E}(m)$ the complete elliptic integral of the second kind [28]. The activation energy as a function of $L$ is shown in Fig. 4. Note that the curve of $\Delta E$ vs. $L$ and its first derivative are both continuous at $L_{c}$; the second derivative, however, is discontinuous, as might be expected of a second-order-like phase transition.

A more profound manifestation of critical behavior at $L_{c}$ is exhibited by the rate prefactor $\Gamma_{0}$. When $L<L_{c}=\pi$, both the stable and saddle states are spatially uniform: $\phi_{\text {stable }}= \pm 1$ and $\phi_{\text {saddle }}=0$. This greatly simplifies the computation of the associated eigenvalues. 


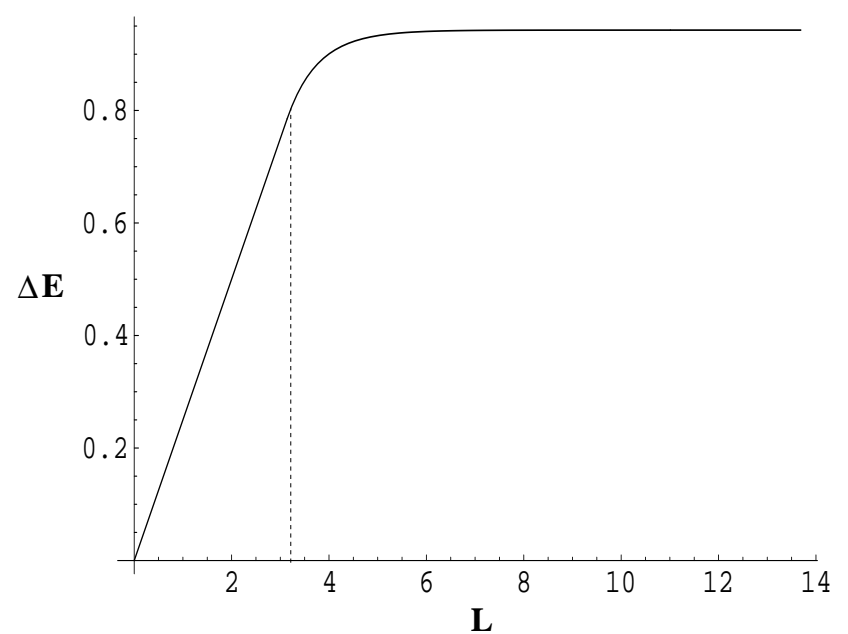

FIG. 4: The activation energy $\Delta E$ as a function of the interval length $L$, for the potential given by Eq. (4) (with all coefficients set equal to one) and Neumann boundary conditions. The dashed line indicates the critical interval length $L_{c}=\pi$ at which the saddle state bifurcation takes place.

It is immaterial which of the two (degenerate) stable states is used; the eigenvalue spectrum is the same at both because of the symmetry under $\phi \mapsto-\phi$. Linearizing around either stable state yields the operator

$$
\Lambda_{s}=-d^{2} / d z^{2}+2
$$

and similarly

$$
\Lambda_{t}=-d^{2} / d z^{2}-1
$$

The eigenvalue spectrum of $\Lambda_{s}$ with Neumann boundary conditions is

$$
\lambda_{s, n}=2+\frac{\pi^{2} n^{2}}{L^{2}} \quad n=0,1,2 \ldots
$$

The eigenvalue spectrum of $\Lambda_{t}$ is similarly

$$
\lambda_{t, n}=-1+\frac{\pi^{2} n^{2}}{L^{2}} \quad n=0,1,2 \ldots
$$

As required, all eigenvalues of $\Lambda_{s}$ are positive, while $\Lambda_{t}$ has a single negative eigenvalue $\lambda_{t, 0}=-1$. Its eigenfunction, which is spatially uniform, is the direction in configuration space along which the optimal escape trajectory approaches $\phi_{t}$.

Putting everything together, we find the Neumann-case rate prefactor when $L<L_{c}$ to be

$$
\begin{aligned}
\Gamma_{0} & =\frac{1}{2 \pi} \sqrt{\frac{\prod_{n=0}^{\infty}\left(2+\frac{\pi^{2} n^{2}}{L^{2}}\right)}{\left|\prod_{n=0}^{\infty}\left(-1+\frac{\pi^{2} n^{2}}{L^{2}}\right)\right|}} \\
& =\frac{1}{2^{3 / 4} \pi} \sqrt{\frac{\sinh (\sqrt{2} L)}{\sin L}} .
\end{aligned}
$$

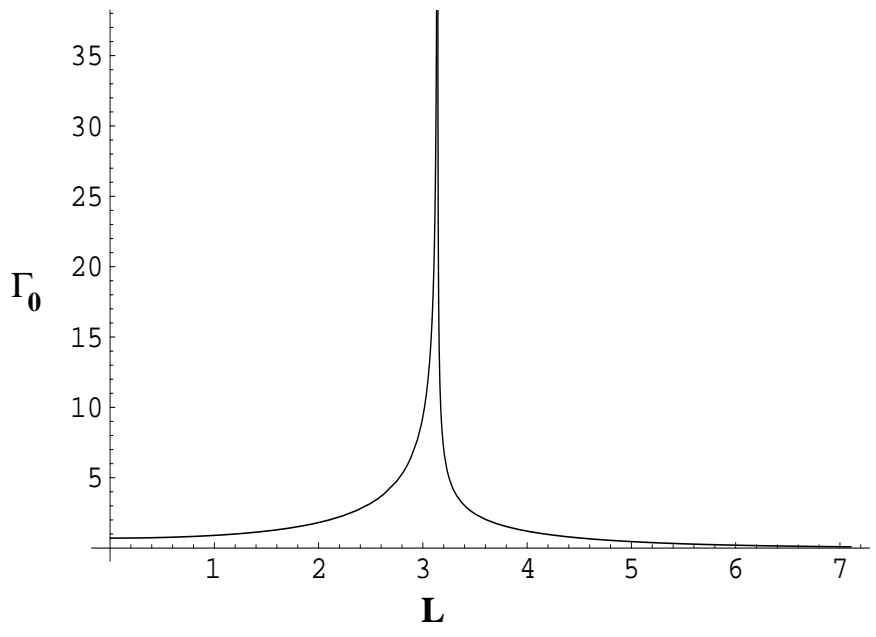

FIG. 5: Rate prefactor $\Gamma_{0}$ for the quartic potential with Neumann boundary conditions, showing the power-law divergence of the prefactor as $L \rightarrow L_{c}^{ \pm}$.

As $L \rightarrow L_{c}^{-}\left(=\pi^{-}\right), \Gamma_{0} \sim\left(L_{c}-L\right)^{-1 / 2}$. This divergence has a simple physical interpretation: the optimal escape trajectory becomes transversally unstable, in the direction defined by the eigenmode $\eta_{1}$, as the critical length is approached. Mathematically the divergence is caused by $\lambda_{t, 1} \rightarrow 0^{+}$as $L \rightarrow L_{c}^{-}$.

When $L>L_{c}$, there are two transition states, namely the nonuniform bounce configurations $\pm \phi_{t}$ given by (15). The associated linearized evolution operator, computed from (11), is

$$
\Lambda_{t}=-\frac{d^{2}}{d z^{2}}-1+\frac{6 m}{1+m} \operatorname{sn}^{2}\left(\frac{x}{\sqrt{m+1}} \mid m\right) .
$$

Calculation of the associated determinant quotient, and the single unstable eigenvalue $\lambda_{t, 0}$, is described in detail in [10], to which the interested reader is referred. The Neumann-case rate prefactor when $L>L_{c}$ is

$$
\begin{array}{r}
\Gamma_{0}=\frac{1}{\pi}\left|1-\frac{2}{1+m} \sqrt{m^{2}-m+1}\right| \\
\times \sqrt{\frac{\sinh (\sqrt{2} L)}{\sqrt{2}|(1-m) \mathbf{K}(m)-(1+m) \mathbf{E}(m)|}} .
\end{array}
$$

As $m \rightarrow 0^{+}\left(L \rightarrow L_{c}^{+}\right), \Gamma_{0} \sim\left(L-L_{c}\right)^{-1 / 2}$. The prefactor over the entire range of $L$ is shown in Fig. 5.

The divergence at $L_{c}$ is striking, but requires interpretation. We defer further discussion to Sec. VII, and turn now to physical applications of the methods and results presented in this section.

\section{TWO APPLICATIONS}

We have established a transition in activation behavior as system length is varied; but is it, and more generally the theory presented above, relevant to actual systems? In this section we briefly discuss two applications. 


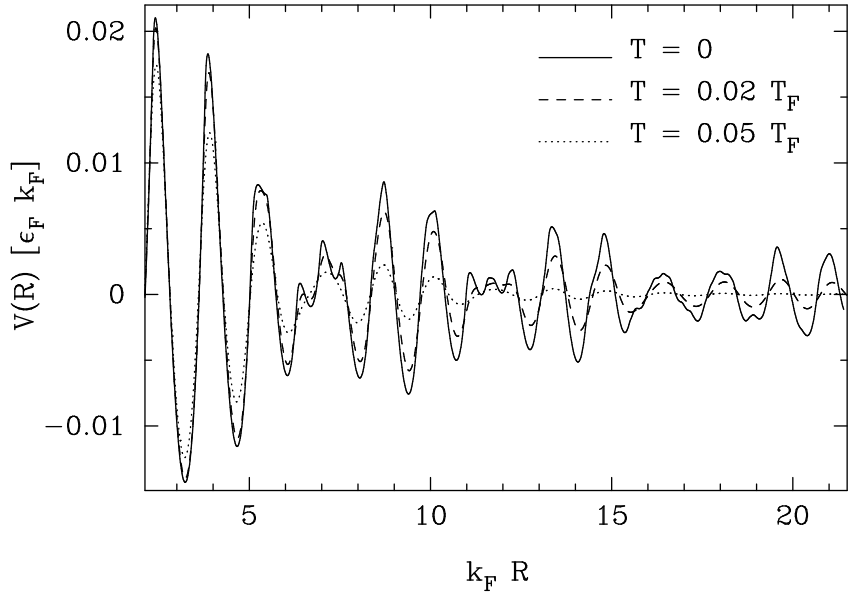

FIG. 6: Electron-shell potential $V_{\text {shell }}(R)$ at three temperatures, computed from the free-electron model of Ref. [36].

\section{A. Lifetimes of monovalent metallic nanowires}

Metallic nanowires are cylindrically-shaped incompressible electron fluids with diameters of order tens of atoms and with lengths hundreds to thousands of atoms. They are stabilized by quantum shell effects [29-31] but at nonzero temperatures are only metastable, with breakup probably due to thermal fluctuations [32-34]. We have proposed [35] a self-consistent continuum approach to studying the lifetimes of monovalent metallic nanowires, with a large-deviationinduced 'collapse' modelled through a stochastic GinzburgLandau field theory, of the kind discussed above. Our theory provides good quantitative agreement with available data on nanowire lifetimes, and accounts for the observed difference in stability between alkali and noble metal nanowires.

We treat a nanowire as a cylinder of length $L$ and radius $R(z)=R_{0}+\phi(z)$, with $z \in[-L / 2, L / 2]$. Radius fluctuations are governed by the stochastic Ginzburg-Landau equation (1), where $V(\phi)$ arises from the zero-temperature electron-shell potential of Fig. 6.

Details of the calculations appear in [35]; here we simply summarize the results, which appear in Table I.

The lifetimes tabulated for sodium nanowires in Table I exhibit a rapid decrease in the temperature interval between $75 \mathrm{~K}$ and $100 \mathrm{~K}$. These lifetimes correlate well with the observed temperature dependence of conductance histograms for sodium nanowires [32-34]. A comparison of the lifetimes of sodium and gold nanowires listed in Table I indicates that gold nanowires are much more stable. In our model this arises from the difference in surface tension: $\sigma_{\mathrm{Au}}=5.9 \sigma_{\mathrm{Na}}$, and is consistent with the observation that noble metal nanowires are much more stable than alkali metal nanowires.

There is also an important prediction contained in Table I, namely that nanowire lifetimes, which exhibit significant variations from one conductance plateau to another, do not vary systematically as a function of radius. It can be seen from Table I that the activation barriers vary by only about $30 \%$ from one plateau to another, and that a wire with a conductance of $96 G_{0}$ has essentially the same lifetime as that with a conductance of $3 G_{0}$. In this sense, the activation barrier variation exhibits universal mesoscopic fluctuations: in any conductance interval, there are very short-lived wires (not shown in Table I) with very small activation barriers, while the longest-lived wires have activation barriers of a universal size:

$$
0<\Delta E_{\infty} \lesssim 0.7\left(\frac{\hbar^{2} \sigma}{m_{e}}\right)^{1 / 2},
$$

The derivation of (25) will be presented elsewhere [37].

At present, lifetimes of wires shorter than $L_{c}$ have not been systematically studied. We expect that over the next several years the technology will improve to the point where this will become possible, and our predictions for a transition in both activation barriers (from barriers essentially independent of length to those varying linearly with length, as in Fig. 4) and prefactors (Fig. 5) can be tested.

\section{B. Magnetic reversal in nanomagnets}

The dynamics of magnetization reversal in submicronsized, single-domain particles and thin films is important for information storage and other magnetoelectronic applications. This problem can be treated with the methods used throughout this paper, but with a more complicated equation of motion than (1). The magnetization dynamics is governed by the Landau-Lifschitz-Gilbert equation [38] perturbed by weak spatiotemporal noise:

$$
\partial_{t} \mathbf{M}=-\gamma\left[\mathbf{M} \times \mathbf{H}_{\mathrm{eff}}\right]+\left(\alpha / M_{0}\right)\left[\mathbf{M} \times \partial_{t} \mathbf{M}\right],
$$

where $M_{0}$ is the magnitude of the magnetization $\mathbf{M}, \alpha$ the damping constant, and $\gamma>0$ the gyromagnetic ratio. The effective field $\mathbf{H}_{\mathrm{eff}}=-\delta E / \delta \mathbf{M}$ is the variational derivative of the total energy $E$, which is given by (with free space permeability $\mu_{0}=1$ ):

$$
\begin{aligned}
E[\mathbf{M}(\mathbf{x})] & =\lambda^{2} \int_{\Omega} d^{3} x|\nabla \mathbf{M}|^{2}+\frac{1}{2} \int_{\mathbf{R}^{3}} d^{3} x|\nabla U|^{2} \\
& -\int_{\Omega} d^{3} x \mathbf{H}_{\mathrm{e}} \cdot \mathbf{M},
\end{aligned}
$$

where $\Omega$ is the region occupied by the ferromagnet, $\lambda$ is the exchange length, and $U$ (defined over all space) satisfies $\nabla \cdot(\nabla U+\mathbf{M})=0$. The first term on the RHS of (27) is the bending energy, the second the magnetostatic energy, and the last the Zeeman energy. We take $\mathbf{H}_{\mathrm{e}} \| \hat{\boldsymbol{\theta}}$, using cylindrical coordinates $(\hat{r}, \hat{\theta}, \hat{z})$. The magnetostatic energy is nonlocal and gives rise to shape anisotropies (for 'soft' magnetic materials, like fcc Co or permalloy, crystalline anisotropies are negligible). The out-of-plane anisotropy energy is especially strong, and forces $\mathbf{M}$ to lie in the plane.

The presence of the nonlocal magnetostatic term complicates analysis. However, the quasi- $2 D$ nature of the problem allows a significant simplification, as shown by Kohn and Slastikov [39]. Their asymptotic scaling analysis applies to 
TABLE I: The lifetime $\tau$ (in seconds) for various cylindrical sodium and gold nanowires at temperatures from $75 \mathrm{~K}$ to $200 \mathrm{~K}$. Here $G$ is the electrical conductance of the wire in units of $G_{0}=2 e^{2} / h, L_{c}$ is the critical length, and $\Delta E_{\infty}$ is the activation energy for an infinitely long wire. From Ref. [35].

\begin{tabular}{|c||c|c|c|c|c||c|c|c|c|c|}
\hline \multicolumn{1}{|c||}{} & \multicolumn{5}{c||}{$\mathrm{Na}$} & \multicolumn{5}{c|}{$\mathrm{Au}$} \\
\cline { 2 - 10 }$G$ & $L_{c}$ & $\Delta E_{\infty}$ & \multicolumn{3}{c|}{$\tau[\mathrm{s}]$} & $L_{c}$ & $\Delta E_{\infty}$ & \multicolumn{3}{c|}{$\tau[\mathrm{s}]$} \\
\cline { 5 - 11 }$\left[G_{0}\right]$ & {$[\mathrm{nm}]$} & {$[\mathrm{meV}]$} & $75 \mathrm{~K}$ & $100 \mathrm{~K}$ & $150 \mathrm{~K}$ & {$[\mathrm{~nm}]$} & {$[\mathrm{meV}]$} & $100 \mathrm{~K}$ & $150 \mathrm{~K}$ & $200 \mathrm{~K}$ \\
\hline \hline 3 & 1.4 & 210 & 30 & $9 \times 10^{-3}$ & $3 \times 10^{-6}$ & 1.5 & 470 & $10^{11}$ & $2 \times 10^{3}$ & 0.2 \\
6 & 2.6 & 170 & 0.06 & $9 \times 10^{-5}$ & $10^{-7}$ & 3.0 & 310 & $10^{3}$ & $7 \times 10^{-3}$ & $2 \times 10^{-5}$ \\
17 & 3.1 & 230 & 500 & 0.08 & $10^{-5}$ & 3.4 & 470 & $9 \times 10^{10}$ & $10^{3}$ & 0.2 \\
23 & 3.7 & 190 & 4 & $2 \times 10^{-3}$ & $10^{-6}$ & 4.1 & 390 & $10^{7}$ & 3 & $2 \times 10^{-3}$ \\
42 & 4.3 & 210 & 50 & 0.01 & $4 \times 10^{-6}$ & 4.8 & 440 & $3 \times 10^{9}$ & 100 & 0.03 \\
51 & 4.5 & 150 & $7 \times 10^{-3}$ & $2 \times 10^{-5}$ & $5 \times 10^{-8}$ & 4.9 & 320 & $2 \times 10^{3}$ & 0.01 & $3 \times 10^{-5}$ \\
96 & 5.8 & 200 & 5 & $2 \times 10^{-3}$ & $10^{-6}$ & 6.3 & 440 & $8 \times 10^{9}$ & 300 & 0.05 \\
\hline
\end{tabular}

the present problem when both the aspect ratio $t / R$ (ring thickness divided by ring mean radius), and $\lambda / R$, are sufficiently small. Because of the high energy cost of variations in $M_{0}$, and because the geometry under consideration admits nonsingular solutions for the vector field $\mathbf{M}, M_{0}$ can be taken to be fixed. The result for the total magnetic energy is then [40]:

$$
\mathcal{E}=\int_{0}^{\ell / 2} d s\left[\left(\frac{\partial \phi}{\partial s}\right)^{2}+\sin ^{2} \phi-2 h \cos \phi\right]
$$

where $\ell$ is related to ring circumference, $h$ is the external magnetic field magnitude, and $\phi$ is the local angle between the in-plane magnetization vector and the local field direction. In (28) energy, length, and field are all dimensionless, normalized by a corresponding characteristic quantity determined by various ring parameters.

Eq. (26) and the variational equation $\mathbf{H}_{\mathrm{eff}}=-\delta E / \delta \mathbf{M}$ yield a nonlinear differential equation that must be satisfied by any time-independent solution:

$$
d^{2} \phi / d s^{2}=\sin \phi \cos \phi+h \sin \phi
$$

There are three 'constant' ( $\phi$ independent of $\theta$ ) but nonuniform $\left(\mathbf{m}=\mathbf{M} / M_{0}\right.$ varies with position) solutions for $0 \leq$ $h<1$ : the stable state $\phi=0(\mathbf{m}=\hat{\theta})$; the metastable state $\phi=\pi(\mathbf{m}=-\hat{\theta})$, and a pair of degenerate unstable states $\phi=\cos ^{-1}(-h)$, which constitute the saddle for a range of $(\ell, h)$. The $\phi=0, \pi$ solutions are degenerate when $h=0$, and the $\phi=\pi$ solution becomes unstable at $h=1$.

We have also found a nonconstant 'bounce' solution of (29), which is the saddle for the remaining range of $(\ell, h)$. It is

$$
\phi(s, m)=2 \cot ^{-1}\left[\operatorname{dn}\left(\frac{s-s_{0}}{\delta} \mid m\right) \frac{\operatorname{sn}(\mathcal{R} \mid m)}{\operatorname{cn}(\mathcal{R} \mid m)}\right],
$$

where $\operatorname{dn}(\cdot \mid m), \operatorname{sn}(\cdot \mid m)$, and $\operatorname{cn}(\cdot \mid m)$ are the Jacobi elliptic functions with parameter $m$ [28], $s_{0}$ is a constant, and $\mathcal{R}$ and $\delta$ are given by

$$
\begin{aligned}
\operatorname{sn}^{2}(\mathcal{R} \mid m) & =1 / m-h / 2 \\
& -(1 / 2 m) \sqrt{m^{2} h^{2}+4(1-m)} \\
\delta^{2} & =\frac{m^{2}}{2-\left(m+\sqrt{m^{2} h^{2}+4(1-m)}\right)} .
\end{aligned}
$$

Imposition of the periodic boundary condition yields a relation between $\ell$ and $m$ :

$$
\ell=2 \mathbf{K}(m) \delta
$$

As $m \rightarrow 0, \operatorname{dn}(x \mid 0) \rightarrow 1$, and the bounce solution reduces to the constant state $\phi=\cos ^{-1}(-h)$. The critical length and field where this occurs are related by

$$
\ell_{c}=\pi \delta_{c}=2 \pi / \sqrt{1-h_{c}^{2}} .
$$

What is interesting here is that the transition is governed by two parameters: not only the length, but also the magnitude of the externally applied magnetic field. While the former cannot be varied continuously, the latter can, allowing for the first time a detailed experimental probe of the transition. Fig. 7 shows theoretical predictions of the magnetization switching rate at two different field strengths for a ring of fixed circumference.

\section{CROSSOVER FROM THERMAL ACTIVATION TO QUANTUM TUNNELING}

Only purely classical activation processes have been considered so far, but the transition in activation behavior as interval length is increased has an interesting parallel with the crossover from classical activation to quantum tunneling as temperature is lowered. The correspondence can be made explicit by mapping system length $L$ in the former case to temperature $T$ in the latter.

The partition function $Z$ at inverse temperature $\beta$ can be written as a Euclidean path integral over trajectories $q(\tau)$, 


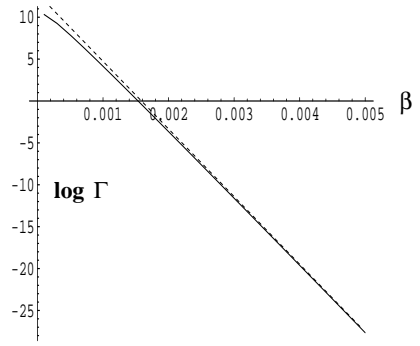

(a)

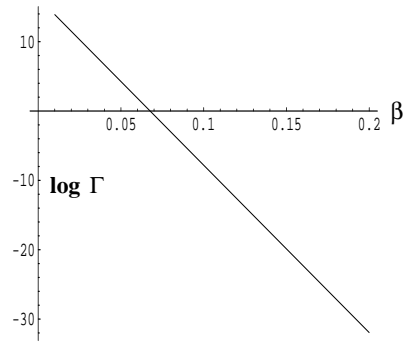

(b)
FIG. 7: Total switching rate (in units of $s^{-1}$ ) vs. inverse temperature $\beta$ (in units of ${ }^{\circ} K^{-1}$ ), at fields of (a) $52.5 \mathrm{mT}$ (nonconstant saddle) and (b) $72.5 \mathrm{mT}$ (constant saddle). Parameters used are $k=.01$, $l=.05, R=200 \mathrm{~nm}, R_{1}=180 \mathrm{~nm}, R_{2}=220 \mathrm{~nm}, M_{0}=8 \times 10^{5} \mathrm{~A} / \mathrm{m}$ (permalloy), $\alpha=.01$, and $\gamma=1.7 \times 10^{11} T^{-1} s^{-1}$. Deviation of lowfield switching rate in (a) from dashed line signals non-Arrhenius behavior. (From Ref. [40].)

where $\tau=$ it is imaginary time, with each path weighted by its Euclidean action $S_{E}$ [41]:

$$
Z=\int \mathcal{D}[q(\tau)] \exp \left\{-S_{E}[q(\tau)] / \hbar\right\}
$$

The integral in (35) runs over all paths periodic in imaginary time, with period $\hbar \beta$.

Eq. (35) is derived from the usual definition of the partition function $Z=\operatorname{Tr}\{\exp [-\beta \mathcal{H}]\}$, where $\mathcal{H}$ includes both the system and its environment. Consequently, even if one is dealing with the tunneling of only a single degree of freedom $q(\tau)$, the effects of friction due to its coupling with the environment must be included. The proper treatment of the effects of damping on quantum mechanical tunneling have been considered by a number of authors; see, for example, [15-18]. Although friction strongly affects the tunneling rate quantitatively, it will not be included here; this is because our only aim is to present the connection between the transition in thermal activation of (infinite-dimensional) classical fields as length is varied, and the crossover from classical activation to quantum tunneling in (one-dimensional) systems as temperature is varied.

An early treatment (that ignored dissipation) was given by Goldanskii [12]. Setting the classical, temperature-dependent Arrhenius factor $\Delta E / k_{B} T$ equal to the zero-temperature quantum tunneling rate through a parabolic barrier, he noted that the characteristic temperature $T_{0}$ for the quantum tunneling $\leftrightarrow$ classical activation crossover was

$$
T_{0}=\hbar \omega_{c} /\left(2 \pi k_{B}\right),
$$

where $\omega_{c}$ is the characteristic frequency of the locally quadratic barrier. Although Goldanskii's approach led only to an estimate, his formula (36) was quite accurate (in the absence of dissipation), as the following more detailed analysis [16-18] will demonstrate (see also [13, 14, 19-22]).

In what follows, we will use the asymmetric potential given by (5). This allows us to consider only incoherent tunneling processes: once the particle tunnels through the barrier, it escapes for good. A double-well potential such as (4) requires consideration of dissipative quantum coherence effects, for which a real-time functional integral approach is better suited (see, for example, [42, 43]). The Euclidean action for a particle of mass $M$ is therefore

$$
\begin{aligned}
S_{E}[q(\tau)] & =\int_{-\beta \hbar / 2}^{\beta \hbar / 2} d \tau\left\{\frac{1}{2} M \dot{q}^{2}(\tau)\right. \\
& \left.+M\left[\frac{\omega_{0}^{2}}{2} q(\tau)^{2}-\frac{\lambda}{3} q(\tau)^{3}\right]\right\}+(\text { frictional terms) }
\end{aligned}
$$

where $\dot{q}$ denotes a derivative with respect to imaginary time $\tau$. In the low-friction limit, extremal paths satisfy

$$
-\ddot{q}(\tau)+\omega_{0}^{2} q(\tau)-\lambda q(\tau)^{2}=0,
$$

with periodic boundary conditions $q(-\beta \hbar / 2)=q(\beta \hbar / 2)$.

Three extremal solutions are physically relevant. The first is the uniform $q(\tau)=0$ solution, which corresponds simply to the stable state at all temperatures. Of the other two, one is uniform:

$$
q_{c,+}(\tau)=\omega_{0}^{2} / \lambda
$$

and the other is a nonuniform bounce:

$$
\begin{aligned}
q_{c,-}(\tau, m) & =\frac{3 \omega_{0}^{2}}{2 \lambda} v(m)^{2} \operatorname{dn}^{2}\left(\frac{\omega_{0} v(m)}{2}\left(\tau-\tau_{0}\right) \mid m\right) \\
& +\frac{\omega_{0}^{2}}{2 \lambda}\left[1-(2-m) v(m)^{2}\right],
\end{aligned}
$$

where as before $0 \leq m \leq 1$ and $v(m)=\left(1-m+m^{2}\right)^{-1 / 4}$. Imposition of the periodic boundary condition relates the parameter $m$ to the inverse temperature:

$$
\beta=4 \mathbf{K}(m) / \hbar \omega_{0} v(m)
$$

The constant $\tau_{0}$ in (40) indicates the translational symmetry in imaginary time arising from the periodic boundary conditions, and corresponds to a zero mode as described in Sec. III.

We can now make explicit the mapping to the thermal activation of classical fields. Here the inverse temperature $\beta$ plays the same role as system length $L$ in Sec. IV. In particular, high temperature corresponds to the small length regime. For $\beta$ smaller than some $\beta_{c}$, we would therefore expect the constant solution $q_{c,+}$ to be the saddle. And indeed it is. The corresponding action is

$$
S_{E}\left[q_{c,+}(\tau)\right]=M \omega_{0}^{6} \beta \hbar /\left(6 \lambda^{2}\right)=\beta \hbar \Delta V,
$$

where $\Delta V=M \omega_{0}^{6} /\left(6 \lambda^{2}\right)$ is the potential energy difference between the potential barrier top and bottom. Consequently, $\exp \left\{-S_{E}\left[q_{c,+}(\tau)\right] / \hbar\right\}=\exp [-\beta \Delta V]$, and the classical Arrhenius factor is recovered.

The bounce solution $S_{E}\left[q_{c,-}(\tau)\right]$ is the saddle at large $\beta$. As in Sec. IV, the transition temperature $T_{c}$ is given by (41) when $m \rightarrow 0^{+}$. We find that $T_{c}=\hbar \omega_{0} /\left(2 \pi k_{B}\right)$, exactly that found by Goldanskii (Eq. (36)), using much simpler arguments. (For the potential used in (37), the curvatures at the top and bottom of the well are equal.) 


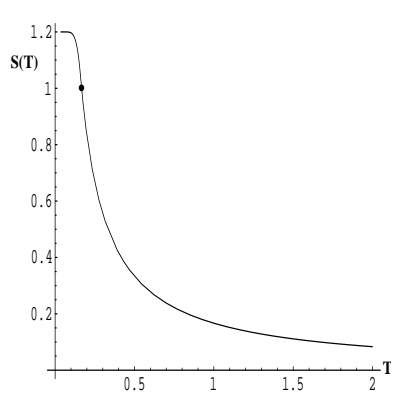

(a)

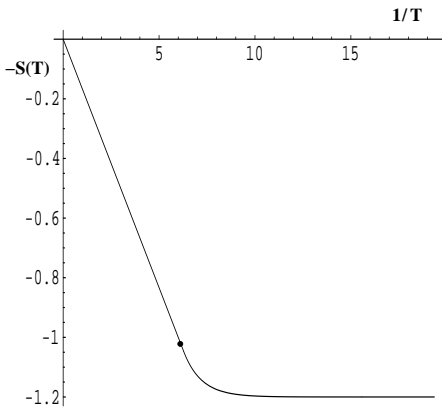

(b)
FIG. 8: (a) The Euclidean action $S_{E}\left[q_{c}(\tau)\right]$ at all temperatures. (b) The logarithm of the leading-order escape rate, shown in an Arrhenius-style plot. In both graphs, $M=\omega_{0}=\lambda=\hbar=k_{B}=1$, and the dot indicates the crossover.

The action in the low-temperature region

$$
\begin{array}{r}
S_{E}\left[q_{c,-}\right]=3 M \omega_{0}^{5} /\left(5 \lambda^{2}\left(1-m+m^{2}\right)^{1 / 4}\right) \\
\times\left[2 \mathbf{E}(m)-\left[(2-m)(1-m) /\left(1-m+m^{2}\right)\right] \mathbf{K}(m)\right] \\
+\left(M \beta \hbar \omega_{0}^{6} / 12 \lambda^{2}\right)\left[1-3(2-m) / 2 \sqrt{1-m+m^{2}}\right. \\
\left.+(2-m)^{3} / 2\left(1-m+m^{2}\right)^{3 / 2}\right]
\end{array}
$$

is, as in the classical field case (cf. Fig. 4), continuous and differentiable at all temperatures; but also as before, its second derivative is discontinuous. The action, along with the leading-order escape rate, is shown in Fig. 8.

The well-known zero-temperature tunneling rate is recovered from (43) in the limit $m \rightarrow 1$. Summarizing, we find that the classical Arrhenius formula is recovered in the hightemperature limit and the quantum tunneling formula is recovered in the zero-temperature limit:

$\exp \left[-S_{E}\left(q_{c,-}\right) / \hbar\right]=\left\{\begin{aligned} \exp \left[-\Delta V / k_{B} T\right] & T \rightarrow \infty \\ \exp \left[-36 \Delta V / 5 \hbar \omega_{0}\right] & T \rightarrow 0\end{aligned}\right.$

while in a narrow region about $T_{0}$ both contribute.

\section{DISCUSSION}

We have demonstrated that in problems involving noiseinduced escape of a classical field over a barrier, a type of second-order phase transition, with what appears to be attendant critical phenomena (such as power-law divergence of the rate prefactor) occurs as one or more external parameters (length of the interval on which the field is defined, external magnetic field if relevant, and so on) is varied. We have also shown that there is a mathematical mapping of this transition to the classical activation $\leftrightarrow$ quantum tunneling transition for a particle escaping a simple potential well. The mapping here involves identifying interval length (and/or magnetic field, if appropriate) in the classical field case to temperature in the classical $\leftrightarrow$ quantum case. To avoid confusion, it should be remembered that the asymptotically small parameter is noise strength (typically, but not necessarily, temperature) in the former problem, and Planck's constant - not temperature - in the latter.

In this section we will address two questions that immediately come to mind: To what extent can the transitions discussed be considered 'real' second-order phase transitions exhibiting critical phenomena; e.g., in the sense that the fluctuations driving the transition occur on all scales? Secondly, how deep is the correspondence between the classical field transition and the quantum $\leftrightarrow$ classical crossover $[13,14,16-22]$ ?

\section{A. Is the phase transition 'real'?}

The short answer is: in a mathematically asymptotic sense yes, but from a strictly (and more physically relevant) criticalphenomena-oriented viewpoint, no. Moreover, the observation of rate prefactor divergence depends crucially on the order in which relevant parameters (temperature, system length, and so on) are varied. There are clearly different saddle configurations, and therefore qualitatively different activation behaviors, on either side of the transition. But the question we are focusing on here is: what is happening very close to the critical lengthscale? This was extensively discussed in [11], and that discussion will be expanded here.

Naively, the transition appears second-order in several respects: the saddle solution is continuous (and even bifurcates in symmetric models such as (4)), and the action is continuous and differentiable at the transition point but has a discontinuous second derivative there. But perhaps most compelling, from the point of view of critical phenomena, is the apparent power-law 'divergence' of the rate prefactor shown in Fig. 5. We therefore examine this in more detail, first asking what it even means for the prefactor to 'diverge'.

Of course, at no lengthscale is the actual prefactor infinite. Consider the analysis of the noisy symmetric GinzburgLandau model given in Sec. IV. It is important to recall that the analysis of the escape rate is, strictly speaking, valid only in an asymptotic sense as $T \rightarrow 0$ : our results apply only to temperatures sufficiently low so that the escape rate is small.

What the formal divergence of the prefactor does mean is that the escape behavior becomes increasingly anomalous as $L_{c}$ is approached, and that it is non-Arrhenius exactly at $L_{c}$, where for all $T \rightarrow 0$ the prefactor is temperature-dependent, scaling as a negative power of $T$. In the region close to $L_{c}$, the rate prefactor $\Gamma_{0}$ is anomalously large, but still finite. The formula (12) — from which the prefactor shown in Fig. 5 was computed - is valid only for $T$ sufficiently small so that the contributions from the quadratic fluctuations about the relevant extremal state of $\mathcal{H}[\phi]$ dominate the action. So as long as all eigenvalues of $\Lambda_{t}$ are nonzero (excluding, if translational symmetry is present, the zero mode which may be extracted), Eq. (12) applies, but only within a temperature region driven to zero as $L \rightarrow L_{c}$. The diminishing size of this region as $L \rightarrow L_{c}$ is controlled by the rate of vanishing of the eigenvalue(s) of smallest magnitude. 
The implication is that the prefactor behavior depends on whether $T \rightarrow 0$ at fixed $L$ near $L_{c}$, or whether $L$ increases (say) through $L_{c}$ at fixed low $T$. In the former case, one will recover Fig. 5. If instead one fixes temperature at some small but nonzero value, one should observe first a rising prefactor as $L$ approaches $L_{c}$, but at some $L$ (depending on $T$ through a type of 'Ginzburg criterion'; cf. Fig. 9 in [11]), the prefactor crosses over to a non-Arrhenius (temperature-dependent) form. As $L$ continues to increase on the other side of $L_{c}$, the sequence of events is reversed.

The procedure of fixing $T$ and varying $L$ is in many instances the more physical one. In this case a correct analysis needs to include higher-order (than quadratic) fluctuations about the transition state, as was done, e.g., in [17]. The next higher-order terms will (unless prevented by symmetry) be nonzero. The behavior remains anomalous, however. Suppose one starts to vary $L$ at fixed $T$, but as soon as non-Arrhenius behavior is encountered, one fixes $L$ and then starts to lower $T$. A subsequent transition from non-Arrhenius to Arrhenius behavior will again be encountered: as $T$ is lowered, the prefactor $\Gamma_{0}$ rises until it reaches the value shown in Fig. 5; it remains constant thereafter.

Lying behind this description is the relative magnitudes of the thermal energy and that due to quadratic fluctuations in the direction of the eigenmode $\eta_{1}$ with vanishing eigenvalue $\lambda_{1}$. Non-Arrhenius behavior should be seen at intermediate' temperatures, where thermal energy is large compared to that due to quadratic fluctuations along the $\eta_{1}$ direction, but small compared to that arising from higher-order fluctuations. 'Intermediate' here depends on $L$, whose closeness to $L_{c}$ determines the magnitude of $\lambda_{1}$. Arrhenius behavior reappears in the 'low' temperature region where the thermal energy is small compared even to that due to the quadratic fluctuations along $\eta_{1}$. And because $\lambda_{1} \rightarrow 0$ as $L \rightarrow L_{c}$, the crossover from non-Arrhenius to Arrhenius behavior occurs at an increasingly lower temperature. Exactly at $L_{c}$, where $\lambda_{1}$ is exactly zero, the escape behavior is non-Arrhenius for all $T \rightarrow 0$.

There's an alternative way of describing the situation: when viewed on 'normal' fluctuation lengthscales of $O\left(T^{1 / 2}\right)$, field fluctuations along the eigenmode direction $\eta_{1}$ appear to be diverging as $L \rightarrow L_{c}$, accompanied by anomalous transition behavior. However, when viewed on an 'anomalous' lengthscale $\left(O\left(T^{1 / 3}\right)\right.$ or $O\left(T^{1 / 4}\right)$ or ..., depending on the form of the potential), those fluctuations remain finite, and one would presumably observe a rounded maximum of the prefactor (when scaled by the appropriate temperature-dependent factor) at $L_{c}$.

The situation is therefore fairly subtle; care must be used to describe the 'transition' in appropriate terms. Moreover, in perhaps the most important respect, the transition fails a central test of criticality - that of the disappearance of a characteristic fluctuational lengthscale. In our problem, even at $L_{c}$ there is a well-defined characteristic lengthscale, albeit an anomalous one.

This is strikingly different from a similar-looking (on the surface) transition described elsewhere [44-46]. This transition in the activation behavior of non-equilibrium systems (e.g., where detailed balance is absent in the zero-noise dynamics) occurs as the result of singularities developing [47] in the action. These singularities lead in turn to the appearance of caustics in the pattern of fluctuational trajectories, as in geometric optics. Here the transition occurs as a parameter in the zero-noise dynamics is varied.

In these systems, fluctuations about the optimal escape trajectory do occur at all scales at the critical point, so one cannot simply 'cure' the divergence by including higher-order terms as before. A de novo scaling theory $[46,47]$ is required. This theory results in an array of nontrivial critical exponents that obeying scaling relations and characterize the divergence (or vanishing) of relevant physical quantities (including, but not limited to, the rate prefactor). Consequently, these nonequilibrium systems can justifiably be said to exhibit true critical behavior at the transition point.

The discussion so far has focused entirely on second-order transitions; what about first-order? This possibility has been discussed by several authors [19-21]. In slightly more complicated classical field theories perturbed by spatiotemporal noise, such as a sixth-degree Ginzburg-Landau model, the nonconstant saddle branch of the energy functional $\mathcal{H}[\cdot]$ can in principle cross the constant saddle branch at a nonzero angle. This should give rise to a first-order transition. So, in the phase plane of these models, the second-order transition point (in the limited sense discussed in this section) is presumably the endpoint of a first-order transition curve.

\section{B. Is the quantum tunneling $\leftrightarrow$ classical activation crossover for a single degree of freedom identical to the transition in} activation behavior of a classical field on finite intervals?

Mathematically, yes, under the following mapping:

\begin{tabular}{ccc} 
& \multicolumn{2}{c}{ Classical $\leftrightarrow$ Quantum } \\
\cline { 2 - 3 } Classical $\phi^{4}$ \\
Tunall parameter & $\hbar$ & $T$ \\
Tunable parameter & $T$ & $L$ \\
Periodic in & $\beta \hbar$ & $L$
\end{tabular}

Here we chose a stochastic Ginzburg-Landau $\phi^{4}$ model for specificity, but one can substitute any of the other classical field theories discussed. For the noisy magnetization dynamics discussed in Sec. V B, it should be remembered that the transition can occur as either length or field is varied. One also need not choose periodic boundary conditions for classical Ginzburg-Landau field theories; the transition will occur in similar fashion (but with fairly minor differences as described in Sec. IV) with other types of boundary conditions. On the other hand, one is constrained to use boundary conditions periodic in $\beta \hbar$ in the quantum $\leftrightarrow$ classical problem.

This mapping is realized when the quantum $\leftrightarrow$ classical transition problem is set up using a Euclidean imaginary-time functional integral formulation, in a potential where incoherent tunneling dominates. The classical problem is approached similarly as a real-space path integral in the limit of weak spatiotemporal noise.

But there is a significant physical difference (leaving aside the obvious ones) between the two classes of problems. In the previous section, we were able to consider - for the classical 
problem - either holding $L$ fixed and lowering $T$, or holding $T$ fixed and moving $L$ through $L_{c}$. In the first case one can, in principle, recover the prefactor divergence shown in Fig. 5, while in the second one should observe a sequence of Arrhenius $\rightarrow$ non-Arrhenius $\rightarrow$ Arrhenius transition behaviors, with the width of the non-Arrhenius region vanishing as $T \rightarrow 0$. 'Non-Arrhenius' means, as usual, a prefactor $\Gamma_{0}$ not determined by (12), and thereby usually aquiring a temperature dependence. In order to see this dependence, one would need to repeat the procedure (fix $T$, move $L$ through the transition) at several different temperatures.

But in the quantum case, there is no such freedom: here the small parameter, $\hbar$, cannot be varied. So a prefactor divergence such as Fig. (5) cannot be observed even in principle.

In a similar vein, the zero mode arising from the use of periodic boundary conditions will differ in the two cases. This mode arises on the nonconstant ('bounce') side of the transition due to translational symmetry - the instanton (or domain wall) separating the two stable solutions can arise anywhere in space (or imaginary time, in the quantum $\leftrightarrow$ classical case). This leads in turn to a $T$-dependent (respectively, $\hbar$-dependent) prefactor for all $L>L_{c}$ (respectively, $T<T_{c}$ ).

It should be kept in mind that we have made the implicit assumption in the classical field case that temperature can be lowered to arbitrarily small values. For many systems this may not be the case: either transition rates will become immeasurably low, or the system itself might undergo a (real) phase change, or new physics may enter in some other way. Nevertheless, the ability to vary temperature does distinguish the classical field transition from the quantum $\leftrightarrow$ classical one. In particular, the magnetic reversal problem (cf. Sec. V B) presents us with the exciting possibility of continuously tuning through the transition by varying an external magnetic field. With the possibility this presents of experimentally studying activation closer to the transition than might otherwise be achieved, we might in this way not only advance our understanding of stochastic reversal in nanoscale magnets, but also uncover new information about the quantum $\leftrightarrow$ classical transition that the constancy of $\hbar$ would otherwise prevent us from obtaining.

\section{ACKNOWLEDGMENTS}

This research was partially supported by the U.S. National Science Foundation Grants No. PHY-0099484 and PHY0351964.
[1] See, for example, J. Garcia-Ojalvo and J.M. Sancho, Noise in Spatially Extended Systems (Springer, New York/Berlin, 1999).

[2] J.S. Langer, Ann. Phys. 41, 108 (1967); Ann. Physics 54, 258 (1969).

[3] L. Néel, Ann. Géophys. 5, 99 (1949).

[4] W. F. Brown, Jr., Phys. Rev. 130, 1677 (1963).

[5] M.C. Cross and P.C. Hohenberg, Rev. Mod. Phys. 65, 851 (1993).

[6] S. Coleman, Phys. Rev. D 15, 2929 (1977); C.G. Callan, Jr. and S. Coleman, Phys. Rev. D 16, 1762 (1977).

[7] G. 't Hooft, Phys. Rev. D 14, 3432 (1976).

[8] C. Morais Smith, B. Ivlev, and G. Blatter, Phys. Rev. B 49, 4033 (1994).

[9] R.S. Maier and D.L. Stein, Phys. Rev. Lett. 87, 270601 (2001).

[10] R.S. Maier and D.L. Stein, in Noise in Complex Systems and Stochastic Dynamics, eds. L. Schimansky-Geier, D. Abbott, A. Neiman, and C. Van den Broeck (SPIE Proceedings Series, v. 5114, 2003), pp. 67-78.

[11] D.L. Stein, J. Stat. Phys. 114, 1537 (2004).

[12] V.I. Goldanskii, Dokl. Acad. Nauk SSSR 124, 1261 (1959); 127, 1037 (1959).

[13] I. Affleck, Phys. Rev. Lett. 46, 388 (1981).

[14] P.G. Wolynes, Phys. Rev. Lett. 47, 968 (1981).

[15] A.O. Caldeira and A.J. Leggett, Phys. Rev. Lett. 46, 211 (1981); Ann. Phys. (NY) 149, 374 (1983); 153, 445 (1983) (Erratum).

[16] A.I. Larkin and Yu.N. Ovchinnikov, JETP 37, 322 (1983).

[17] H. Grabert and U. Weiss, Phys. Rev. Lett. 53, 1787 (1984).

[18] P.S. Riseborough, P. Hänggi, and E. Freidkin, Phys. Rev. A 32, 489 (1985)

[19] E.M. Chudnovsky, Phys. Rev. A 46, 8011 (1992).

[20] A.N. Kuznetsov and P.G. Tinyakov, Phys. Lett. B 406, 76 (1997).

[21] D.A. Gorokhov and G. Blatter, Phys. Rev. B 56, 3130 (1997).
[22] K.L. Frost and L.G. Yaffe, Phys. Rev. D 59, 065013 (1999).

[23] L.S. Schulman, Techniques and Applications of Path Integration (Wiley, New York, 1981), chapter 29.

[24] P. Hänggi, P. Talkner, and M. Borkovec, Rev. Mod. Phys. 62, 251 (1990)

[25] Theory of Continuous Fokker-Planck Systems, edited by F. Moss and P. V. E. McClintock (Cambridge University Press, Cambridge, 1989).

[26] A.J. McKane and M.B. Tarlie, J. Phys. A 28, 6931 (1995).

[27] H. Kleinert and A. Chervyakov, Phys. Lett. A 245, 345 (1998).

[28] Handbook of Mathematical Functions, edited by M. Abramowitz and I. A. Stegun (Dover, New York, 1965).

[29] F. Kassubek, C.A. Stafford, H. Grabert, and R.E. Goldstein, Nonlinearity 14, 167 (2001)

[30] C.-H. Chang, F. Kassubek, and C.A. Stafford, Phys. Rev. B 68 , 165614 (2003).

[31] D.F. Urban and H. Grabert, Phys. Rev. Lett. 91, 256803 (2003).

[32] A.I. Yanson, I.K. Yanson, and J.M. van Ruitenbeek, Nature 400, 144 (1999).

[33] A.I. Yanson, I.K. Yanson, and J.M. van Ruitenbeek, Phys. Rev. Lett. 84, 5832 (2000).

[34] A.I. Yanson, J.M. van Ruitenbeek, and I.K. Yanson, Low Temp. Phys. 27, 807 (2001).

[35] J. Bürki, C.A. Stafford, and D.L. Stein, in Noise in Complex Systems and Stochastic Dynamics II, eds. Z. Gingl, J. Sancho, L. Schimansky-Geier, and J. Kertesz, (SPIE Proceedings Series, v. 5471, 2004), pp. 367-379.

[36] C.A. Stafford, D. Baeriswyl, and J. Bürki, Phys. Rev. Lett. 79, 2863 (1997).

[37] J. Bürki, C.A. Stafford, and D.L. Stein, in preparation.

[38] F.H. deLeeuw, R. van den Doel, and U. Enz, Rep. Prog. Phys. 43, 689 (1980).

[39] R.V. Kohn and V.V. Slastikov, "Another Thin-Film Limit of Mi- 
cromagnetics", Arch. Rat. Mech. Anal., in press. Available at http://www.math.nyu.edu/faculty/kohn.

[40] Kirsten Martens, D.L. Stein, and A.D. Kent, "Magnetic Reversal in Mesoscopic Ferromagnetic Rings", submitted to Phys. Rev. Lett.; available as cond-mat/0410561.

[41] R.P. Feynman, Statistical Mechanics (Benjamin, Reading, MA, 1972).

[42] A.J. Bray and M.A. Moore, Phys. Rev. Lett. 49, 1545 (1982).
[43] S. Chakravarty and A.J. Leggett, Phys. Rev. Lett. 52, 5 (1984).

[44] R.S. Maier and D.L. Stein, Phys. Rev. Lett. 71, 1783 (1993).

[45] M.I. Dykman, M.M. Millonas, and V.N. Smelyanskiy. Phys. Lett. A 195, 53 (1994).

[46] R.S. Maier and D.L. Stein, J. Stat. Phys. 83, 291 (1996).

[47] R.S. Maier and D.L. Stein, Phys. Rev. Lett. 85, 1358 (2000). 